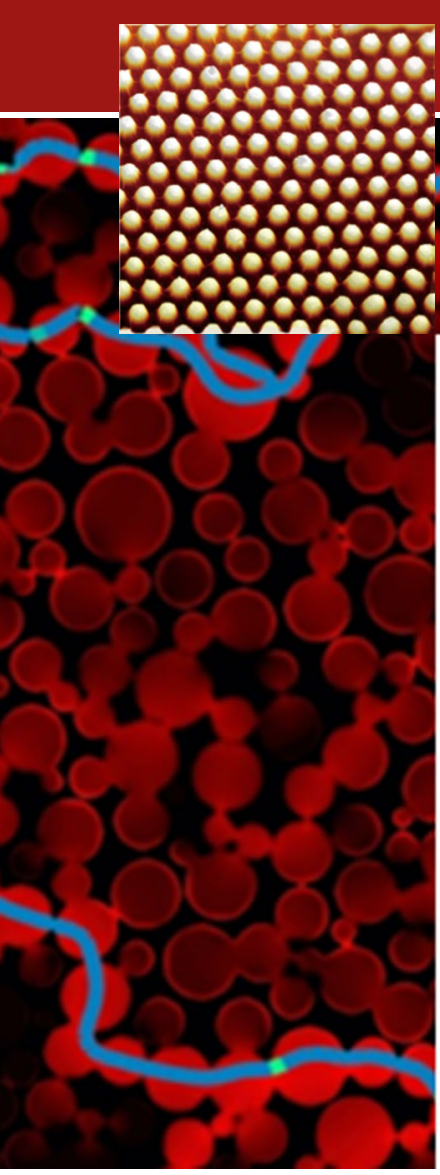

\title{
Electric-field-assisted processing of ceramics: Nonthermal effects and related mechanisms
}

\author{
Olivier Guillon, Roger A. De Souza, Tarini Prasad Mishra, and \\ Wolfgang Rheinheimer
}

Field-assisted processing methods, such as spark plasma sintering and flash sintering, have considerably expanded the toolbox of ceramic engineering. Depending on the conditions, substantial electric currents may flow through the material resulting in fast heating rates due to Joule heating. Here, we focus on nonthermal effects induced by electric fields during processing of fluorite- and perovskite-based ceramics. The fundamentals of how a field can directly modify defect formation and migration in crystals are discussed. In addition, the interplay of ion transport and electrical conductivity is considered, this interplay being crucial to understanding nonthermal effects caused by electric fields (as in memristive switching). Electrochemical reactions leading to new phases or reduction are also described, as are densification rates and sintering parameters that are significantly affected even though the sample temperature is held constant. Finally, as grain-boundary properties and segregation are changed by ion transport, we describe how both retardation and acceleration of grain growth can be achieved including graded microstructures.

\section{Introduction}

In this article, we focus on the theoretical prediction and the experimental identification of phenomena induced by electric fields of different magnitudes. Macroscopic Joule heating of the sample needs to be excluded or at least well controlled in order to deconvolute nonthermal from thermal effects, the latter being covered in this issue. ${ }^{1}$ Large thermal effects are present in flash sintering (thermal runaway, possibly transient melting of necks), ${ }^{2,3}$ but electric-field-assisted processing of ceramics should not be reduced to flash sintering only. A series of different studies, which will be detailed later on, has shown that both $\mathrm{AC}$ and DC fields offer an additional degree of freedom to synthesize materials, to tailor microstructures and properties through acceleration or retardation of reactions, independent control of densification kinetics, and grain size.

The materials considered in this article are ionic oxides based either on the fluorite structure $\left(\mathrm{MO}_{2}\right.$ such as $\mathrm{ZrO}_{2}$ and $\left.\mathrm{CeO}_{2}\right)$ or on the perovskite structure $\left(\mathrm{ABO}_{3}\right.$, such as $\left.\mathrm{SrTiO}_{3}\right)$. According to Reference 5 one-half of all papers published on flash sintering focus on these materials. These specific classes of polycrystalline materials are found in a broad range of applications (from electrochemical devices such as solid-oxide fuel cells, gas separation membranes, and sensors to storage elements such as memristors, multilayer ceramic capacitors over mechanical components). ${ }^{6-8}$

In the following, fundamentals of how a field can affect defect formation and migration in crystals are described. They are complemented by specific sections covering electrochemical reactions, sintering, and grain-boundary mobility under electric fields.

\section{Fundamentals}

The mobility of an ion in an ionic solid, $u_{\text {ion }}$, can be expressed as

$$
u_{\text {ion }}=u_{\text {def }} \frac{c_{\text {def }}}{c_{\text {ion }}}
$$

where $u_{\text {def }}$ is the mobility of the point defect responsible for ion mobility and $c_{\text {def }}$ and $c_{\text {ion }}$ are the concentration of those defects and of the ions in question (and $c_{\text {def }} \ll c_{\text {ion }}$ by definition). We thus recognize that, for ion transport to take place, point defects are required to be sufficiently mobile $\left(u_{\text {def }}\right)$ and 
to be present at sufficiently high concentrations $\left(c_{\text {def }}\right)$. For a polycrystalline ceramic body, we also need to be aware that various paths may be available for ion transport - through the bulk phase, along grain boundaries, or along free surfaces. Since $u_{\text {def }}$ and $c_{\text {def }}$ will in general differ considerably for these different paths, $u_{\text {ion }}$ also will. Next, we consider $u_{\text {ion }}$ first in the bulk and second along grain boundaries.

Ionic solids consist of two or more sublattices, and generally, the ions of one sublattice are more mobile than the ions of the other sublattice(s). Fluorite-structured $\mathrm{CeO}_{2}$ and perovskitestructured $\mathrm{SrTiO}_{3}$ are both characterized by $u_{\text {anion }} \gg u_{\text {cation. }}$. Consequently, processes such as sintering in these materials are governed by cation transport. The low $u_{\text {cation }}$ is due to the corresponding $u_{\text {def }}$ and $c_{\text {def }}$ both being very low. Increasing $u_{\text {cation }}$ to a sufficient level (e.g., for densification) is usually achieved through raising the temperature, since both $u_{\text {def }}$ and $c_{\text {def }}$ increase exponentially with increasing temperature. The temperature dependence of $u_{\mathrm{def}}$ is described in terms of the activation enthalpy of defect migration, $\Delta H_{\mathrm{mig}}^{\ddagger}$ :

$$
u_{\mathrm{def}}=\frac{A}{T} \mathrm{e}^{-\Delta H_{\mathrm{mig}}^{\ddagger} / k_{\mathrm{B}} T},
$$

For cation-vacancy migration in $\mathrm{CeO}_{2}$ and $\mathrm{SrTiO}_{3},{ }^{10}$ $\Delta H_{\text {mig }}^{\ddagger} \sim 4 \mathrm{eV}$. Hence, to increase $u_{\text {def }}$ by $10^{5}$ at $T=1273 \mathrm{~K}$, one has to raise the temperature by $400 \mathrm{~K}$. Returning briefly to Equation 1, and taking $\mathrm{CeO}_{2}$ as our example, we note that $c_{\text {ion }}$ is the concentration of cerium ions and $c_{\mathrm{def}}$ is the concentration of cerium vacancies or cerium interstitials. Such cation defects (vacancies, interstitials) are generated through the Schottky and Frenkel disorder reactions, which for $\mathrm{CeO}_{2}$ are written in Kröger-Vink notation ${ }^{11}$ as follows:

$$
\begin{aligned}
& \mathrm{Ce}_{\mathrm{Ce}}^{\times}+2 \mathrm{O}_{\mathrm{O}}^{\times} \rightleftharpoons \mathrm{v}_{\mathrm{Ce}}^{\prime \prime \prime \prime}+2 t e x t \ddot{\mathrm{O}}+\mathrm{CeO}_{2}(\operatorname{surf}) K_{\mathrm{Sch}}=\left[\mathrm{v}_{\mathrm{Ce}}^{\prime \prime \prime \prime}\right]\left[\mathrm{v}_{\mathrm{O}}\right]^{2}, \\
& \mathrm{Ce}_{\mathrm{Ce}}^{\times}+\mathrm{v}_{i}^{\times} \rightleftharpoons \mathrm{v}_{\mathrm{Ce}}^{\prime \prime \prime \prime}+\mathrm{Ce}_{i}^{\cdots \cdots} K_{\mathrm{Fr}}=\left[\mathrm{v}_{\mathrm{Ce}}^{\prime \prime \prime \prime}\right]\left[\mathrm{Ce}_{\mathrm{i}}^{\cdots \cdot \cdot}\right],
\end{aligned}
$$

where the $K$ 's refer to equilibrium constants and $[X]$ is the concentration of the defect $X$. The difference between these two intrinsic disorder reactions is that Schottky disorder can only occur at an (internal or external) interface, after which the defects generated can diffuse into the bulk, whereas Frenkel can occur directly within the bulk phase. If we assume that the defect chemistry is governed by acceptor dopants fixing the concentration of oxygen vacancies, we find

$$
\left[\mathrm{v}_{\mathrm{Ce}}^{\prime \prime \prime \prime}\right]=\frac{K_{\mathrm{Sch}}}{\left[\mathrm{v}_{\mathrm{O}}\right]} \propto \mathrm{e}^{-\frac{\Delta H_{\mathrm{Sch}}}{k_{\mathrm{B}} \mathrm{T}}},
$$

and

$$
\left[\mathrm{Ce}_{\mathrm{i}}^{\cdots \cdots}\right]=\frac{K_{\mathrm{Fr}}}{K_{\mathrm{Sch}}}\left[\mathrm{v}_{\mathrm{O}}\right] \propto \mathrm{e}^{-\frac{\Delta H_{\mathrm{Fr}}-\Delta H_{\mathrm{Sch}}}{k_{\mathrm{B}} \mathrm{T}}} .
$$

For $\mathrm{CeO}_{2}$, calculated values ${ }^{12}$ are $\Delta H_{\mathrm{Sch}} \sim 6.5 \mathrm{eV}$ and $\Delta H_{\mathrm{Fr}} \sim 12 \mathrm{eV}$.
Can an electric field $E$ directly alter $u_{\text {def }}$ and $c_{\text {def }}$ ? The answer in both cases is: yes, it is possible. Next, we discuss the mechanisms, focusing on cation transport (for the reason previously given), but analogous results apply to anion transport.

The standard model describing $u_{\text {def }}(E, T)$ considers the field to decrease $\Delta H_{\mathrm{mig}}^{\ddagger}$ for forward jumps and increase it for backward jumps, resulting in ion jumps in the forward direction occurring far more frequently and those in the opposite direction far less frequently. ${ }^{13}$ A simple, qualitative treatment (see Reference 14 for a quantitative prediction) yields for an ion of charge ze jumping a distance $2 a$,

$$
u_{\text {def }} \propto \frac{1}{E} \exp \left(-\frac{\Delta H_{\mathrm{mig}}^{\ddagger}}{k_{\mathrm{B}} T}\right) \sinh \left(\frac{|z| e a E}{k_{\mathrm{B}} T}\right) .
$$

At low fields, the sinh term reduces to its argument, and $u_{\mathrm{def}}$ is, as required, independent of $E$; at high fields $\left[E>\left(k_{\mathrm{B}} T\right) /(|z| e a)\right], u_{\mathrm{def}}$ becomes dependent on $E$. Enormous fields are required, however: To increase $u_{\text {def }}$ for tetravalent cations by $10^{5}$ at $T=1273$, one has to apply ${ }^{14}$ a field of $E \approx 1.97 \times 10^{9} \mathrm{Vm}^{-1}$.

The mechanism through which a field generates Frenkel pairs (to increase $c_{\text {def }}$ ) is the ionic, classical variant of the Zener effect in semiconductors (the latter involving electrons quantum mechanically tunneling from the valence band to the conduction band to generate electron-hole pairs). In the (classical) case of Frenkel-pair generation, a regular ion moves a distance $a_{\mathrm{vi}}$ from its lattice site to an interstitial site, leaving a vacancy behind. A thermodynamic approach ${ }^{15}$ relates the relevant quantities to the field required: $E \sim \Delta H_{\mathrm{Fr}} /\left(|z| e a_{\mathrm{vi}}\right)$. For Frenkel-pair generation in $\mathrm{CeO}_{2}, E \sim 10^{10} \mathrm{Vm}^{-1}$.

It has been shown that phonon modes close to the Brillouin zone edge can generate Frenkel defects in oxides. ${ }^{16}$ This would provide an alternative mechanism, but at present, it is not clear how a field produces these phonon modes in an ionic crystal, nor is it clear how high the field has to be for this mechanism to be operative.

We now consider $u_{\text {ion }}$ along grain boundaries (free surfaces being qualitatively similar). The perturbed structure of a grain boundary will clearly affect $\Delta H_{\text {mig }}^{\ddagger}$ and thus $u_{\text {def }}$. For highly mobile oxide ions, the perturbation is evidently detrimental. For the less mobile cations, the perturbation could well be beneficial. ${ }^{2}$ Nevertheless, $E \sim 10^{9} \mathrm{Vm}^{-1}$ will still be required at $T=1273$ to produce a huge enhancement in $u_{\text {ion }}$ (since atomic distances and atomic barriers at interfaces do not differ by orders of magnitude from bulk values).

The perturbed structure at grain boundaries also alters the thermodynamics of defect formation. Differences in the Gibbs formation energies of defects between bulk and interface $\left(\Delta G_{\text {seg }}\right)$ drive the formation of space-charge zones. In acceptor-doped $\mathrm{CeO}_{2}$ and $\mathrm{SrTiO}_{3}, \Delta G_{\text {seg }}$ for oxygen vacancies drives the formation of space-charge zones in which oxygen vacancies are depleted, ${ }^{17-20}$ acceptor cations are accumulated, and cation vacancies are accumulated. Cation mobility along 
grain boundaries can thus be strongly accelerated within the space-charge zones because $c_{\text {def }}$ is much higher, ${ }^{21}$ and experimental data for cation diffusion in these materials are consistent with this picture. Applying a field to modify $\Delta G_{\text {seg, },}$, and thus the effective $c_{\text {def }}$, has been argued $^{18}$ to require $E \sim 10^{10} \mathrm{Vm}^{-1}$, but quantitative simulation studies are necessary to confirm this.

There is also the intriguing possibility of modifying $\Delta G_{\text {seg }}$ by changing the atomistic structure. Applying a field was found to change the interface structure of a $\mathrm{SrTiO}_{3}$ twist-bicrystal, but the interfaces of two bicrystals with the same nominal twist angle were compared rather than those of two identical bicrystals. ${ }^{22}$ Further experimental and theoretical work is also necessary here.

We thus conclude that according to current understanding, there are no plausible mechanisms by which a field can directly accelerate the migration or the generation of cation defects (or of anion defects) at room temperature and higher at field strengths $E<10^{7} \mathrm{Vm}^{-1}$ [this being the lowest limit of $\left.\left(k_{\mathrm{B}} T\right) /(|z| e a)\right]$.

\section{Electrochemical gradients}

In this section, we will discuss the influence of the electric field on electrochemical reactions and resulting modification of the material properties.

The electrochemical blackening of perovskite-based titanates ${ }^{23}$ and zirconia ${ }^{24}$ provides an example of internal solid-state reactions driven by a DC voltage. The boundary conditions (blocking or nonblocking electrodes for one type of charge carrier, possible exchange with oxygen in the atmosphere) affect the electrochemically induced internal reaction mechanisms and the formation of $n$ - and $p$-type conducting regions. ${ }^{25}$ As a consequence, gradients in the electrical conductivity develop in the specimen. Electrons and holes can recombine with a light emission deviating from the black-body radiation. ${ }^{25}$ The morphology of the moving boundary can be predicted as function of the transport kinetics of the material itself. ${ }^{24}$ As grain boundaries act as barriers for oxygen ion transport, polycrystalline ceramic specimens show significantly slower blackening rates than single-crystalline specimens under identical conditions.

As an example shown in Figure 1, blackening of dense polycrystalline $10 \mathrm{~mol} \%$ gadolinium-doped ceria (GDC) was initiated from the cathode side and advanced toward the anode side. ${ }^{26}$ The observed chromatic alteration of the sample is due to the migration of the positively charged oxygen vacancies $\left(v_{\mathrm{O}}\right)$ that accumulated at the cathode side, while the oxygen ions moved to the anode side. The nonlinear rise in conductivity occurs when the sample is completely blackened. Ex situ x-ray photoelectron spectroscopy (XPS) of the specimen revealed a partial remnant reduction which enhances the electronic conductivity of the material:

$$
\mathrm{O}_{\mathrm{O}}^{\times} \rightarrow 2 \mathrm{e}^{\prime}+\ddot{\mathrm{v}_{\mathrm{O}}}+\frac{1}{2} \mathrm{O}_{2} .
$$

Furthermore, shift in the valence-band edge and reduction of the bandgap of the specimen was confirmed by diffuse reflectance measurements. ${ }^{26}$

This polarity-induced electrochemical reduction results in a conductivity gradient through the specimen from one electrode to the other, generating thermal inhomogeneity in the sample. By utilizing AC electric field at higher frequency,${ }^{27}$ the temperature gradient could be reduced. Additionally, the nature of the electrode material and its ability to exchange oxygen ions could generate gradient in the electrochemical properties in the specimen. This can be overcome by selecting appropriate electrode materials. ${ }^{27}$

The electric field-induced oxygen vacancy migration could induce nanoscale redox reaction as previously discussed, forming a so-called switching filament. It is now widely accepted that the resistive switching phenomenon as in redox-based memristive devices is caused by an internal redistribution of oxygen vacancies within a switching filament. ${ }^{28}$

In ionic solids, electric field not only induces migration of oxygen vacancies (faster migrating species), but also influence the migration of cations (slower migrating species), when an electrical bias (DC) is applied for longer period of time. The applied DC bias may induce compositional gradients due to differences in the cations mobility in ternary compounds. This type of inhomogenization is termed "kinetic" unmixing and has been investigated for $\mathrm{NiTiO}_{3}{ }^{29}$ Above a given voltage, the perovskite even starts to decompose. These phenomena can directly affect the material properties that will eventually degrade the performance and lifetime of the functional ceramic-based device. However, related works on the field-induced migration of cations are 
very limited as compared to the field-induced migration of oxygen ion.

\section{Sintering}

Sintering involves several thermally activated, densifying, and nondensifying mechanisms. The initial stage of sintering leading to compact consolidation is usually dominated by surface diffusion-with no significant shrinkage. It seems that moderate electric fields do not modify this early-stage sintering, as shown for yttrium-stabilized zirconia (YSZ) and GDC. ${ }^{30,31}$ However, both AC and DC fields lead to measurable change in the densification kinetics of the intermediate stage of sintering. ${ }^{32-34}$

Systematic investigations were carried out for two yttriumdoped ceria compositions to thoroughly quantify electric fieldinduced effects. The discontinuous sinter-forging technique was applied for the first time to measure sintering parameters under AC electric fields, well below the flash-sintering conditions as predicted by the thermal runaway model. ${ }^{32}$ The combination of sample temperature measurement by thermocouples and thermoelectric finite element simulations ensured that the macroscopic Joule heating effect was excluded. ${ }^{35}$

Electric fields increase shrinkage in both axial and radial directions, without inducing a measurable microstructural anisotropy. The sintering (or viscous) parameters as defined within the continuum mechanical description of sintering ${ }^{35}$ are a function of both temperature and microstructure. The uniaxial/shear viscosity measures the resistance of a sintering body to deformation under uniaxial/shear stress. The viscous Poisson's ratio describes the deformation of a material in directions perpendicular to the direction of loading. As shown in Figure 2, these parameters are significantly changed by an external electric field. However, the sintering trajectory (i.e., mean grain size as function of relative density) was similar for all conditions. ${ }^{36}$ The argument of grain-growth retardation under electric field (as observed on fully sintered YSZ samples) ${ }^{37}$ is therefore not valid here and cannot be taken as responsible for the observed changes in sintering parameters.

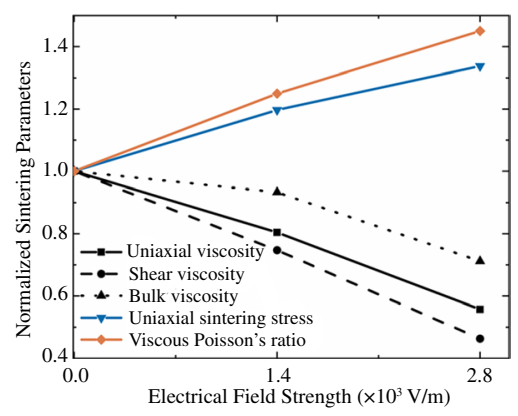

Figure 2. Normalized sintering parameters measured for $10 \mathrm{~mol} \%$ yttrium-doped ceria as function of electric field (relative density of $80 \%$ and constant sample temperature of $1210^{\circ} \mathrm{C}$ ).
The sintering stress - which is the thermodynamic driving force for densification - increases almost linearly with the applied electric field whereas uniaxial, bulk, and shear viscosities, which determine densification kinetics, decrease. Symmetry between tension and compression is confirmed also under electric field. ${ }^{38}$ In addition, viscous Poisson's ratio increases with relative density and electrical fields. These changes can be attributed to easier grain-boundary sliding under electric field. Bulk viscosity is indeed inversely proportional to the grain-boundary diffusion coefficient and shear viscosity depends on both grain-boundary diffusion coefficient and grain-boundary sliding. ${ }^{39}$ The activation energies related to grain-boundary diffusion seem to decrease with increasing electrical field strengths. ${ }^{31}$ The observed softening of the sintering material under electric field should be beneficial to the constrained sintering of layers.

Several reasons can be proposed to rationalize these experimental results: gradual modification of the grain-boundary structure and associated space-charge layer, or temperature microgradients, which are not detected at the macroscopic scale. In support of the first assumption, amorphous films at grain boundaries were observed in pure zirconia, resulting from the severe reducing condition owing to the DC electric field during the flash-sintering process. ${ }^{40}$ As evidenced by impedance spectroscopy, grain-boundary conductivity and permittivity were significantly higher in flash-sintered samples, while the bulk properties did not vary much. ${ }^{41}$ For the latter explanation, temperature gradients may develop-at least transiently due to high current density achieved at the interparticle contact areas and stimulate thermodiffusion. ${ }^{42}$ Using numerical models based on dielectric physics, the local field strength at interparticle necks was estimated to be orders of magnitude higher than the externally applied field, possibly leading to local dielectric breakdown ${ }^{43}$ and Joule heating.

\section{Grain growth in electric field}

Grain growth reduces the grain-boundary area of polycrystals and is generally a thermally activated process. Assuming curvature-driven grain growth, the mean grain size $R$ follows the equation $R^{2}-R(t=0)^{2}=k t$. The growth rate $k=2 \alpha m_{\mathrm{GB}} \gamma_{\mathrm{GB}}$ includes the grain-boundary energy $\gamma_{\mathrm{GB}}$, the grain-boundary mobility $m_{\mathrm{GB}}$, and a geometrical constant $\alpha . m_{\mathrm{GB}}$ is known to depend on electric fields since the $1970 \mathrm{~s},{ }^{44}$ but our understanding is still incomplete, partially due to the experimental complexity.

In $\mathrm{SrTiO}_{3}$, recent model experiments used blocking electrodes to prevent current-induced Joule heating and to isolate nonthermal effects. ${ }^{45,46}$ The growth of single-crystalline seeds into polycrystals was observed with well-controllable driving forces for grain growth. ${ }^{47}$ An electric field $\left(\leq 5 \times 10^{4} \mathrm{Vm}^{-1}\right)$ was applied perpendicular to the growth direction. Gradients in the growth rate of the seed crystal were observed: faster growth of the seed crystal occurred at the negative electrode, while the positive electrode showed a growth rate comparable to field-free experiments (Figure 3a). 
a

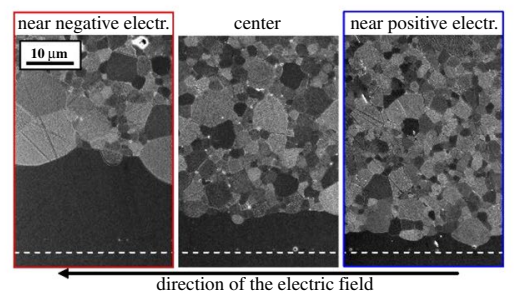

C

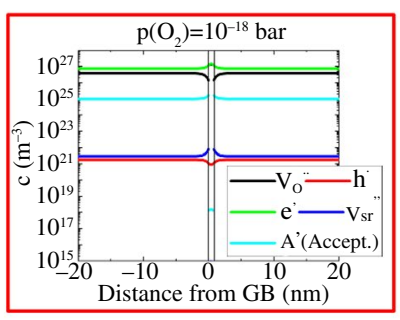

Figure 3. Graded microstructure evolution at $1460^{\circ} \mathrm{C}$ after $2 \mathrm{~h}$ with $5 \times 10^{4} \mathrm{~V} \mathrm{~m}^{-1}$ applied (a). Field-induced $\mathrm{v}_{\mathrm{O}}$ transport resulting in local oxidation and reduction (b). Point defect concentrations as resulting from space at the grain boundaries in reduced (c) and oxidized $\mathrm{SrTiO}_{3}$ (d). b

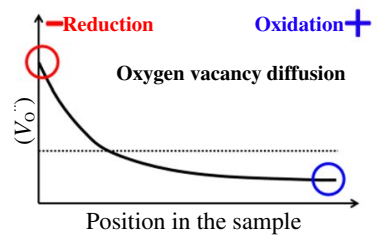

d

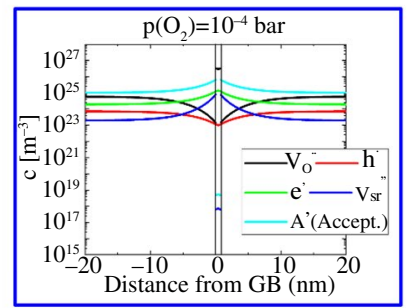

This change of the growth rate by the electric field can be understood by a field-driven migration of $\mathrm{v}_{\mathrm{O}}$ toward the negative electrode. In perovskites, $D\left(\mathrm{v}_{\mathrm{O}} \cdots, 1400^{\circ} \mathrm{C}\right) \approx 10^{-4} \mathrm{~cm}^{2} \mathrm{~s}^{-1}$ ${ }^{48}$ allowing migration of $\mathrm{v}_{\mathrm{O}}$ across the sample within seconds. Accordingly, the oxide becomes reduced (oxidized) at the negative (positive) electrode (Figure 3b). Grain boundaries in oxides generally feature space charge,${ }^{49}$ where the grainboundary core charge depends on the oxidation state. For $\mathrm{SrTiO}_{3}$, this dependence is well understood, allowing to calculate the point defect concentrations for reducing (Figure 3c) and oxidizing (Figure 3d) conditions.

The cationic segregation to the space charge is impacting $m_{\mathrm{GB}}$ (solute drag): Grain-boundary migration is coupled to the migration of segregated defects and, thus, is coupled to their diffusion kinetics. In $\mathrm{SrTiO}_{3}$, the diffusion of $\mathrm{Sr}$ is much slower than oxygen $\left(D_{\mathrm{Sr}} \approx 10^{-19} \mathrm{~cm}^{2} \mathrm{~s}^{-1}\right.$ at $\left.1400^{\circ} \mathrm{C}\right),{ }^{50}$ and cationic dopants are likely in the same range or slower. Accordingly, grain-boundary migration is dragged by the diffusion of cationic segregation. ${ }^{47,51}$ As segregation depends on the oxygen partial pressure (Figure 3c-d), the drag effect of segregation depends on the reduction state: in reduced $\mathrm{SrTiO}_{3}$, little cationic segregation to the grain boundaries is evident. Consequently, $m_{\mathrm{GB}}$ is much higher than for oxidized $\mathrm{SrTiO}_{3}{ }^{52}$

These relations between local reduction state and cationic segregation allow understanding the field effect on $m_{\mathrm{GB}}$ : at the negative electrode, the oxide is partially reduced by the drift of $\mathrm{v}_{\mathrm{O}}$. Less space charge and cationic segregation is evident there resulting in less diffusional drag and a higher $m_{\mathrm{GB}}$ at the negative electrode (and vice versa at the positive electrode).
While $\mathrm{SrTiO}_{3}$ is the only example where the impact of electric fields on $m_{\mathrm{GB}}$ is known to this detail, there are other materials as barium titanate..$^{53}$ and zirconia $^{54,55}$ with similar field effects on microstructure evolution.

Similar observations were made for flash sintering of $\mathrm{SrTiO}_{3}{ }^{56}$ However, flash sintering involves currents and Joule heating so that an interplay with thermal effects on microstructure evolution is likely. Still, the impact of electric fields on grain growth can be understood from an electrochemical perspective and gradients in the microstructure pose a powerful tool to evaluate field effects on material processing.

\section{Summary}

Clear nonthermal effects induced by electric fields have been observed during well-controlled sintering and graingrowth experiments. For example, the progressive reduction under electric field leading to mixed ionic electronic conductivity is the key for rationalizing the incubation time before flash sintering is triggered. ${ }^{25,26,57} \mathrm{~A}$ reduction from $\mathrm{Ce}^{4+}$ to $\mathrm{Ce}^{3+}$ can positively affect the densification kinetics of ceria and enhance grain growth, as shown by sintering studies carried out in reducing atmosphere. ${ }^{58,59}$ These nonthermal effects are independent from thermal effects, enabling to use electric fields as additional parameter to synthesize materials and manufacture components under conditions far from equilibrium. Benefits are, among others, shortened sintering times and decreased sintering temperatures, tailored microstructures with controlled grain-size gradients, and superplastic forging of dense ceramic parts.

Remaining points to clarify are local temperature and electric potential distributions in powder compacts, resulting grain-boundary phases. For this, characterization methods with high spatial and temporal resolution are required as well as in situ techniques in order to characterize the dynamics of field-assisted sintering. ${ }^{60}$ Finally, multiscale modeling is required to relate processes at the atomic scale to the macroscopic behavior of material.

Such a better understanding does not only open new strategies to synthesize and process ceramic materials, but also enables to improve the reliability of devices based on solid-state ionics such as SOFCs. For example, pores and gas-bubble migration has been observed in YSZ at temperatures as low as $800^{\circ} \mathrm{C}$ (i.e., operating temperature of SOFCs) ${ }^{61}$ This phenomenon, attributed to the parallel surface diffusion of cations along the field and oxygen ion diffusion against the field, has to be considered when designing and operating such cells. 


\section{Acknowledgments}

The authors acknowledge funding from the German Science Foundation (DFG), under priority program "Fields Matter" SPP 1959 [BR 3418/1, DE 2854/9, GU 993/9; GU 993/10; HO 1165/20] and under the Emmy-Noether-Program (RH 146/1).

\section{Funding}

Open Access funding enabled and organized by Projekt DEAL.

\section{Open Access}

This article is licensed under a Creative Commons Attribution 4.0 International License, which permits use, sharing, adaptation, distribution and reproduction in any medium or format, as long as you give appropriate credit to the original author(s) and the source, provide a link to the Creative Commons licence, and indicate if changes were made. The images or other third party material in this article are included in the article's Creative Commons licence, unless indicated otherwise in a credit line to the material. If material is not included in the article's Creative Commons licence and your intended use is not permitted by statutory regulation or exceeds the permitted use, you will need to obtain permission directly from the copyright holder. To view a copy of this licence, visit http://creativecommons.org/licenses/by/4.0/.

\section{References}

1. G.M. Jones, M. Biesuz, W. Ji, S. Fisher John, C. Grimley, C. Manière, C.E.J. Dancer, MRS Bull. 46 (1), 59 (2021)

2. M. Biesuz, V.M. Sglavo, J. Eur. Ceram. Soc. 39, 115 (2019)

3. R. Chaim, G. Chevallier, A. Weibel, C. Estournès, J. Appl. Phys. 121, 145103 (2017)

4. O. Guillon, C. Elsässer, 0. Gutfleisch, J. Janek, S. Korte-Kerzel, D. Raabe, C.A. Volkert, Mater. Today 21, 527 (2018)

5. M. Yu, S. Grasso, R. Mckinnon, T. Saunders, M.J. Reece, Adv. Appl. Ceram. 116, 24 (2017)

6. A.J. Moulson, J.M. Herbert, Electroceramics: Materials, Properties, Applications (Wiley, New York, 2003)

7. Advanced Ceramics for Energy Conversion and Storage, 1st ed. https://www. elsevier.com/books/advanced-ceramics-for-energy-conversion-and-storage/ guillon/978-0-08-102726-4

8. V. Rana, R. Waser, in Memristors and Memristive Systems, R. Tetzlaff, Ed. (Springer, New York, 2014), pp. 223-251

9. S. Beschnitt, T. Zacherle, R.A. De Souza, J. Phys. Chem. C. 119, 27307 (2015)

10. V. Metlenko, A.H.H. Ramadan, F. Gunkel, H. Du, H. Schraknepper, S. HoffmannEifert, R. Dittmann, R. Waser, R.A. De Souza, Nanoscale 6, 12864 (2014)

11. F.A. Kröger, H.J. Vink, in Solid State Physics, F. Seitz, D. Turnbull, Eds. (Academic Press, New York, 1956), vol. 3, pp. 307-435

12. T. Zacherle, A. Schriever, R.A. De Souza, M. Martin, Phys. Rev. B 87, 134104 (2013)

13. E.J.W. Verwey, Physica 2, 1059 (1935)

14. A.R. Genreith-Schriever, R.A. De Souza, Phys. Rev. B 94, 224304 (2016)

15. M. Schie, S. Menzel, J. Robertson, R. Waser, R.A. De Souza, Phys. Rev. Mater. 2, 035002 (2018)

16. M. Jongmanns, D.E. Wolf, J. Am. Ceram. Soc. 103, 589 (2020)

17. A. Tschöpe, S. Kilassonia, R. Birringer, Solid State Ionics 173, 57 (2004)

18. R.A. De Souza, E.C. Dickey, Philos. Trans. R. Soc. Lond. A 377, 20180430 (2019)

19. R.A. De Souza, Phys. Chem. Chem. Phys. 11, 9939 (2009)

20. D.S. Mebane, R.A. De Souza, Energy Environ. Sci. 8, 2935 (2015)

21. J.P. Parras, R.A. De Souza, Acta Mater. 195, 383 (2020)

22. L.A. Hughes, K. van Benthem, J. Am. Ceram. Soc. 102, 4502 (2019)

23. T. Baiatu, R. Waser, K.-H. Härdtl, J. Am. Ceram. Soc. 73, 1663 (1990)

24. J. Janek, C. Korte, Solid State Ionics 116, 181 (1999)
25. R. Kirchheim, Solid State Ionics $\mathbf{3 2 0} 239$ (2018)

26. T.P. Mishra, R.R.I. Neto, G. Speranza, A. Quaranta, V.M. Sglavo, R. Raj, O. Guillon, M. Bram, M. Biesuz, Scr. Mater. 179, 55 (2020)

27. M. Biesuz, L. Pinter, T. Saunders, M. Reece, J. Binner, V.M. Sglavo, S. Grasso, Materials (2018). https://doi.org/10.3390/ma11071214

28. C. Baeumer, C. Schmitz, A.H.H. Ramadan, H. Du, K. Skaja, V. Feyer, P. Müller, B. Arndt, C.-L. Jia, J. Mayer, R.A. De Souza, C. Michael-Schneider, R. Waser, R. Dittmann, Nat. Commun. 6, 8610 (2015)

29. J. Chun, M. Martin, H.-I. Yoo, J. Appl. Phys. 117, 124504 (2015)

30. M. Cologna, R. Raj, J. Am. Ceram. Soc. 94, 391 (2011)

31. C. Cao, PhD thesis, Forschungszentrum Jülich $\mathrm{GmbH}$, Verlag Zentralbibliothek, Jülich (2020). https://juser.fz-juelich.de/record/884793

32. H. Conrad, D. Yang, Mater. Sci. Eng. A 559, 591 (2013)

33. M. Biesuz, D. Rizzi, V.M. Sglavo, J. Am. Ceram. Soc. 102, 813 (2019)

34. T.B. Holland, T.B. Tran, D.V. Quach, U. Anselmi-Tamburini, J.R. Groza, A.K. Mukherjee, J. Eur. Ceram. Soc. 32, 3675 (2012)

35. R.K. Bordia, G.W. Scherer, Acta Metall. 36, 2393 (1988)

36. C. Cao, R. Mücke, O. Guillon, Acta Mater. 182, 77 (2020)

37. D. Yang, H. Conrad, Scr. Mater. 63, 328 (2010)

38. C. Cao, Y. Sasaki, R. Mücke, K. Morita, 0. Guillon, Scr. Mater. 187, 137 (2020)

39. C. Cao, R. Mücke, F. Wakai, O. Guillon, Scr. Mater. 178, 240 (2020)

40. N. Morisaki, H. Yoshida, T. Kobayashi, T. Tokunaga, T. Yamamoto, J. Am. Ceram. Soc. 101, 3282 (2018)

41. J.-C. M'Peko, J.S.C. Francis, R. Raj, J. Am. Ceram. Soc. 96, 3760 (2013)

42. E.A. Olevsky, L. Froyen, J. Am. Ceram. Soc. 92, S122 (2009)

43. T.B. Holland, U. Anselmi-Tamburini, D.V. Quach, T.B. Tran, A.K. Mukherjee, J. Eur. Ceram. Soc. 32, 3659 (2012)

44. W.D. Kingery, J. Am. Ceram. Soc. 57, 1 (1974)

45. W. Rheinheimer, M. Fülling, M.J. Hoffmann, J. Eur. Ceram. Soc. 36, 2773 (2016)

46. W. Rheinheimer, J.P. Parras, J.-H. Preusker, R.A. De Souza, M.J. Hoffmann, J. Am. Ceram. Soc. 102, 3779 (2019)

47. W. Rheinheimer, M. Bäurer, C.A. Handwerker, J.E. Blendell, M.J. Hoffmann, Acta Mater. 95, 111 (2015)

48. R.A. De Souza, V. Metlenko, D. Park, T.E. Weirich, Phys. Rev. B 85, 174109 (2012)

49. G. Gregori, R. Merkle, J. Maier, Prog. Mater Sci. 89, 252 (2017)

50. K. Gömann, G. Borchardt, M. Schulz, A. Gömann, W. Maus-Friedrichs, B. Lesage, 0. Kaïtasov, S. Hoffmann-Eifert, T. Schneller, Phys. Chem. Chem. Phys. 7, 2053 (2005)

51. K.S.N. Vikrant, W. Rheinheimer, H. Sternlicht, M. Bäurer, R.E. García, Acta Mater. 200, 727 (2020)

52. W. Rheinheimer, M. Bäurer, M.J. Hoffmann, Acta Mater. 101, 80 (2015)

53. W. Rheinheimer, X.L. Phuah, H. Wang, F. Lemke, M.J. Hoffmann, H. Wang, Acta Mater. 165, 398 (2019)

54. Y. Dong, H. Wang, I.-W. Chen, J. Am. Ceram. Soc. 100, 876 (2017)

55. Y. Dong, I.-W. Chen, J. Am. Ceram. Soc. 101, 1058 (2018)

56. H.-R. Jin, S.-H. Yoon, J.-H. Lee, J.-H. Lee, N.M. Hwang, D.-Y. Kim, J.-H. Han, J. Am. Ceram. Soc. 87, 1747 (2004)

57. N. Masó, A.R. West, Chem. Mater. 27, 1552 (2015)

58. V. Esposito, D.W. Ni, Z. He, W. Zhang, A.S. Prasad, J.A. Glasscock, C. Chatzichristodoulou, S. Ramousse, A. Kaiser, Acta Mater. 61, 6290 (2013)

59. T.P. Mishra, A.M. Laptev, M. Ziegner, S.K. Sistla, A. Kaletsch, C. Broeckmann, 0. Guillon, M. Bram, Materials 13, 3184 (2020)

60. X.L. Phuah, J. Cho, T. Tsakalakos, A.K. Mukherjee, H. Wang, X. Zhang, MRS Bull. 46 (1), 44 (2021)

61. I.W. Chen, S.W. Kim, J. Li, S.J. Kang, F. Huang, Adv. Energy Mater. 11, 1383 (2012)

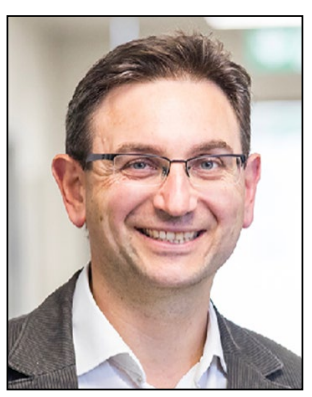

Olivier Guillon has been a director at the Institute of Energy and Climate Research—Materials Synthesis and Processing (IEK-1, Forschungszentrum Julich), Germany, and a professor at the RWTH Aachen University, Germany, since 2014. He established an Emmy Noether Group at the Technical University of Darmstadt, Germany, and received a first professorship at the University of Jena, Germany. He currently is coordinating the DFG-funded priority program, "Fields Matter" (Manipulation of Matter Controlled by Electric and Magnetic Fields: Toward Novel Synthesis and Processing Routes of Inorganic Materials). Guillon is a member of the World Academy of Ceramics and the International Institute for the Science of Sintering. Guillon can be reached by email at o.guillon@fz-juelich.de. 


\section{ELECTRIC-FIELD-ASSISTED PROCESSING OF CERAMICS: NONTHERMAL EFFECTS AND RELAIED MECHANISMS}

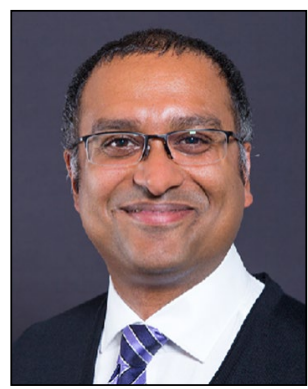

Roger A. De Souza has been a researcher at the Institute of Physical Chemistry at RWTH Aachen University, Germany, since 2002. He received his BEng degree in 1992, and his $\mathrm{PhD}$ degree in 1996 from Imperial College, UK. He completed postdoctoral research at Karlsruhe University, Germany, and at the Max Planck Institute for Solid State Research, Germany. His research includes both experimental and computational approaches, on complex oxides for energy and information technologies. His current focus is characterizing and understanding transport processes in these oxides and at their interfaces. De Souza can be reached by email at

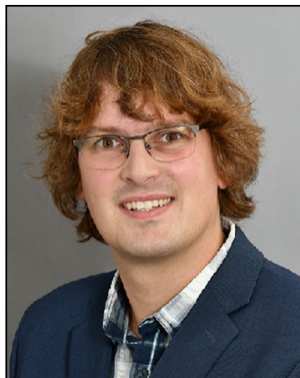

Wolfgang Rheinheimer leads an Emmy Noether Group funded by the German Research Foundation, Germany. He received his Diploma at the Karlsruhe Institute of Technology (KIT), Germany. He completed postdoctoral research at KIT, and was a visiting professor at Purdue University. His research focuses on microstructure evolution in ionic materials, and the segregation and processing aspects during sintering and grain growth and the resulting functional properties. Rheinheimer can be reached by email at w.rheinheimer@fz-juelich.de.

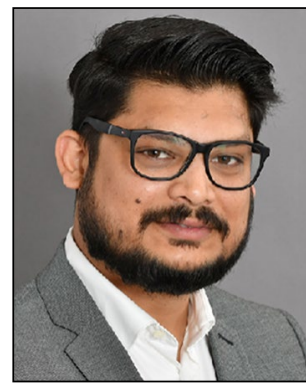

Tarini Prasad Mishra is a postdoctoral researcher in the Materials Synthesis and Processing (IEK-1) Group at Forschungszentrum Jülich, Germany. He received his MSc degree in materials science from the Technical University of Darmstadt, Germany, in 2016, and his PhD degree from Ruhr University, Bochum, Germany, in 2020 , within the DFG priority program SPP 1959 "Fields Matter." His research focuses on understanding flash sintering and unleashing its potential. He is the recipient of the 2019 Acta Student Award. Mishra can be reached by email at t.mishra@fz-juelich.de. 